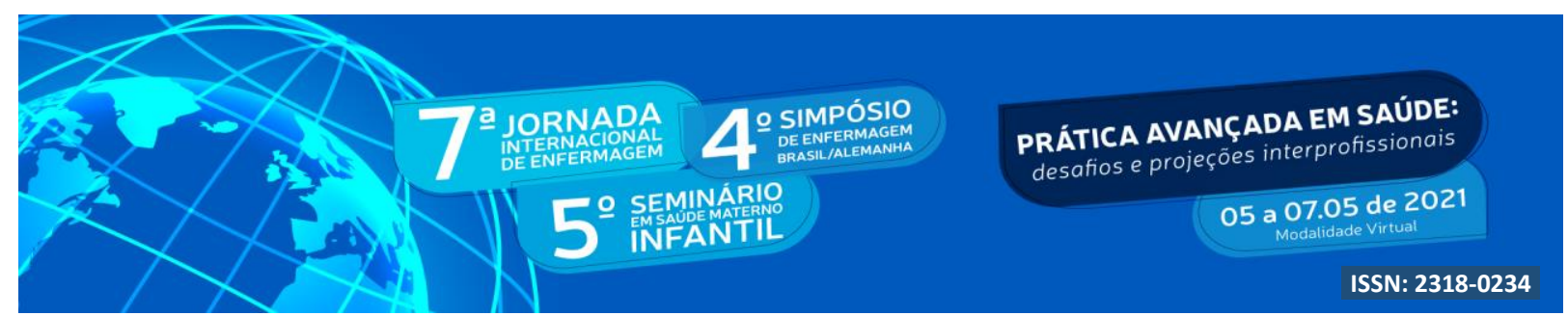

DOI: http://doi.org/10.48195/jie2021-033

\title{
DESAFIOS PARA CONSOLIDAÇÃO DA AMAMENTAÇÃO EM UNIDADE DE TERAPIA INTENSIVA NEONATAL: REVISÃO NARRATIVA DE LITERATURA ${ }^{1}$
}

\author{
Gloria Cogo ${ }^{2}$; Gabrieli Ronzani ${ }^{3}$; Flávia Camef Dorneles Lenz ${ }^{4}$; Lenise Dutra da Silva ${ }^{5}$.
}

\begin{abstract}
RESUMO
Este estudo teve como objetivo conhecer as publicações acerca da amamentação do prematuro na Unidade de Terapia Intensiva Neonatal. Trata-se de uma revisão narrativa de literatura com busca na Base de Dados de Enfermagem e na Literatura Latino Americana e do Caribe Em Ciências da Saúde, no mês de abril de 2020. Utilizou-se a análise temática de conteúdo de Minayo. A partir da busca e aplicação dos critérios de inclusão e exclusão, foram analisados quatro artigos. A partir da aplicação da análise de conteúdo emergiram três categorias: A importância do aleitamento materno na prematuridade; Fatores que influenciam no desfecho da amamentação em UTIN e Os profissionais da equipe e seu papel para manutenção da amamentação em UTIN. Os resultados mostraram diversos desafios enfrentados durante esse processo e a importância da equipe de enfermagem como mediadora desse percurso.
\end{abstract}

Palavras-chave: Aleitamento Materno; Prematuridade; Unidade de Terapia Intensiva Neonatal.

\begin{abstract}
This study aimed to know the publications about breastfeeding the premature infant in the Neonatal Intensive Care Unit. This is a narrative literature review with a search in the Nursing Database and in Latin American and Caribbean Literature in Health Sciences, in the month of April 2020. Minayo's thematic content analysis was used. From the search and application of the inclusion and exclusion criteria, four articles were analyzed. From the application of content analysis, three categories emerged: The importance of breastfeeding in prematurity; Factors that influence the outcome of breastfeeding in the NICU and the professionals of the team and their role in maintaining breastfeeding in the NICU. The results showed several challenges faced during this process and the importance of the nursing team as a mediator of this path.
\end{abstract}

Keywords: Breastfeeding; Prematurity; Neonatal Intensive Care Unit.

\footnotetext{
${ }^{1}$ Revisão de Literatura

${ }^{2}$ Acadêmica do curso de Enfermagem da Universidade Regional integrada do Alto Uruguai e das Missões URICâmpus de Santiago. E-mail: gloriabio1@gmail.com

${ }^{3}$ Acadêmica do curso de Enfermagem Universidade Regional integrada do Alto Uruguai e das Missões URICâmpus de Santiago. E-mail: gabrielironzani.gr@gmail.com

${ }^{4}$ Acadêmica do curso de Enfermagem Universidade Regional integrada do Alto Uruguai e das Missões URICâmpus de Santiago. E-mail: flaviacamefd@gmail.com

${ }^{5}$ Enfermeira. Mestre em Saúde Materno Infantil. Docente do Curso de Enfermagem da Universidade Regional integrada do Alto Uruguai e das Missões URI-Santiago E-mail: enfermeira.lenise@gmail.com
} 


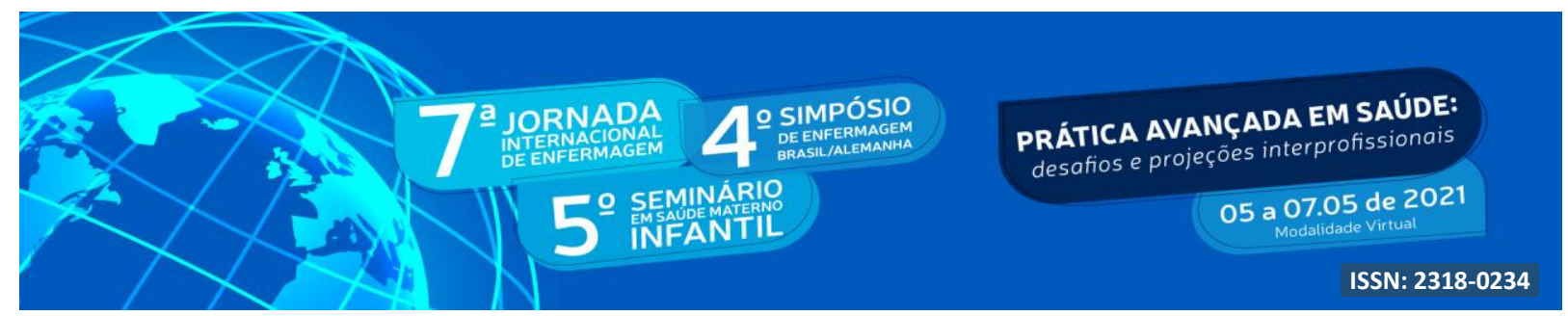

\section{INTRODUÇÃO}

Diversos são os fatores que levam a uma gestação de alto risco, tais com idade materna, antecedentes de prematuridade, sangramento vaginal no segundo semestre, entre outros. A prematuridade é considerada um fator de risco para a mortalidade neonatal, um recém-nascido pré-termo (RNPT) apresenta cerca de 50 vezes mais chances de risco de morte se comparado a recém-nascidos a termo. (TRONCO, et al 2015).

Para os Recém-Nascidos ( $\mathrm{RN})$ prematuros, o leite materno oferece inúmeros benefícios. Sendo eles, a menor incidência e gravidade de enterocolite necrosante, sepse e retinopatia da prematuridade, promove ainda, a ampliação no desempenho neuropsicomotor, e fortalece o vínculo mãe-filho, contribuindo para um menor tempo de hospitalização e menor incidência de reinternações (LIMA, et al 2019).

Em decorrência de internações UTIN, o contato físico do RN com a mãe tornase limitado. A partir de então, surgem os sentimentos como de frustração, insegurança, preocupação, ansiedade e falta de confiança na capacidade de cuidar do seu filho e a participação da mãe na assistência é mínima, somados à necessidade de suportes como oxigênio, ventilação mecânica ou outras vias alternativas de alimentação. Esses fatores afetam diretamente o processo do aleitamento materno na UTIN (MORAIS, GUIRARDI, MIRANDA, 2020).

\section{OBJETIVO}

Este estudo tem como objetivo conhecer as publicações acerca da amamentação do prematuro na unidade de terapia intensiva neonatal (UTIN).

\section{METODOLOGIA}

Este estudo trata-se de uma Revisão Narrativa de Literatura (RNL), acerca do conhecimento produzido sobre a importância da amamentação na prematuridade e os fatores que influenciam nesse processo. O período de coleta ocorreu em abril de 2020, as bases de 


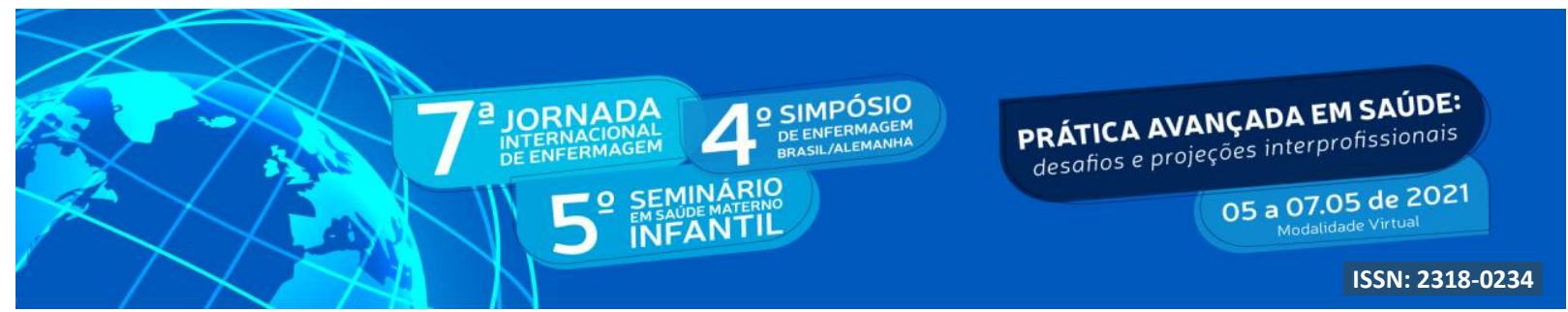

dados utilizadas foram a Base de Dados de Enfermagem (BDENF) e Literatura Latino Americana e do Caribe Em Ciências da Saúde (LILACS). Como descritores foram utilizados "prematuridade"; "aleitamento materno"; "uti neonatal", associados com o operador boleano AND.

A partir da busca surgiram 12 produções científicas na BDENF e 24 produções na LILACS, aplicaram-se os critérios de inclusão, selecionando somente os trabalhos completos disponíveis nas bases de dados, com pesquisas relacionadas ao tema proposto, nos idiomas português, inglês ou espanhol, que estivessem dentro do recorte temporal de 2015 a 2020.

Excluíram-se as produções que não apresentam texto completo e não contemplavam a temática, além de dissertações e teses. Os estudos repetidos nas bases de dados foram contabilizados apenas uma vez. Com isso, foram selecionados quatro artigos para análise.

Para análise dos resultados qualitativos utilizou-se à análise de conteúdo temática de Minayo, que se estende em três fases. A primeira fase é a pré-análise que se trata da escolha das produções a serem analisadas, a segunda fase se dá pela exploração das produções, codificando e classificando os dados apresentados, e a terceira etapa refere-se à fase do tratamento dos resultados obtidos e a interpretação com criação das categorias dos estudos apresentados (MINAYO, 2014).

\section{RESULTADOS E DISCUSSÃO}

Quanto à caraterização dos dados quantitativos e qualitativos dos artigos analisados destaca-se que dos quatro artigos 100\% são estudos qualitativos, destes tantos eram 10\% estudos descritivos, 10\% transversal e 10\% abordagem fenomenológica. Os estudos apresentam como cenário a Unidade de Terapia Intensiva Neonatal (UTIN), considerando suas condições necessárias atender o RN estado grave em virtude de prematuridade ou intercorrências perinatal, além disso, os participantes foram os membros da equipe multiprofissional e a família.

Quanto ao ano de publicação verificaram-se três (3) artigos do ano de 2015, e uma (1) publicação do ano de 2017. Quanto à procedência da produção destaca-se que os quatro estudos foram realizados no Brasil, e as produções ocorreram uma no Rio Grande do Sul, uma no estado do Ceará, uma em São Paulo, e uma no estado do Paraná. 


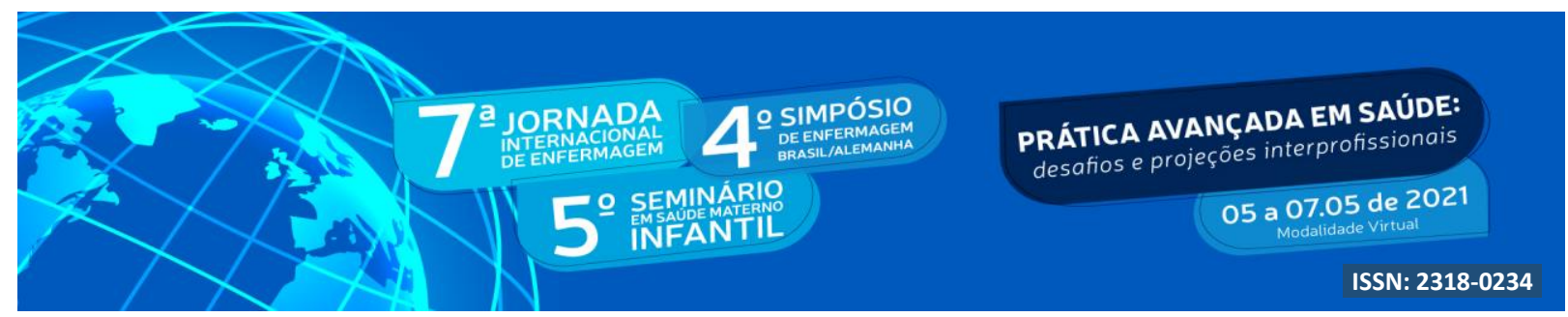

A partir da análise emergiram três categorias: A importância do aleitamento materno na prematuridade; Fatores que influenciam no desfecho da amamentação em UTIN e Os profissionais da equipe e seu papel para manutenção da amamentação em UTIN.

\subsection{A importância do aleitamento materno na prematuridade}

O nascimento de um filho prematuro para algumas mães pode transformar a maternidade em algo não excepcional, indo na contramão do simbolismo social que a maternidade carrega. Visto que, a hospitalização do RN em uma UTIN traz consigo o afastamento desta mãe do convívio social e familiar, além de estar submetidos à rotina estressante de uma terapia intensiva, presenciando diariamente procedimentos dolorosos e invasivos realizados no neonato, e concomitante a isso sentimentos de impotência, insegurança e incerteza (TRONCO, et al 2015).

Em meio a este cenário surge uma prática fundamental e cheia de desafios a amamentação, inúmeros são os benefícios que o aleitamento materno promove, segundo Cruz e Sebastião (2015), além de fortalecer as relações afetivas entre mãe e bebê, contribui para a consolidação da autoconfiança da nutri em poder cuidar do RN. Para a saúde da criança destaca ainda, contribuições para o crescimento e desenvolvimento craniofacial e motor-oral do bebê, colaborando assim, para prevenção de alterações do sistema estomatognático.

Outros benefícios podem ser citados, tais como, a proteção às mulheres do câncer de mama, o baixíssimo custo financeiro, e o auxílio na redução de chances de uma gravidez não planejada. Ademais, o AM exerce grande influência na redução de mortalidade de lactentes, pois possui anticorpos fortalecendo o sistema imunológico, é facilmente absorvido, facilitando o transito gastrintestinal e ainda, reduz a possibilidade de icterícia neonatal (UEMA, et al 2015). Somando-se a isso, em um estudo se destaca que o leite da mãe de um prematuro possui algumas substâncias como lipídeos e proteínas, sendo na quantidade necessária para a situação em que o RN se encontra (BEZERRA, et al 2015).

A amamentação auxilia ainda na retomada da relação mãe-filho, muitas vezes prejudicada em meio à internação neonatal. Por meio da manutenção da lactação é possível nutrir o RN e ao mesmo tempo incluir a mãe no cuidado de seu filho (TRONCO, et al 2015). 


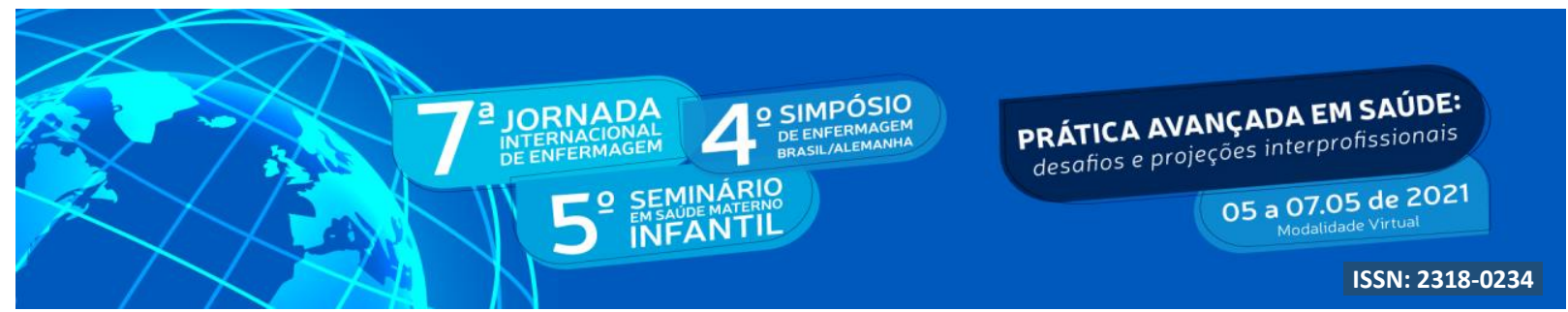

Enfatizando dessa forma, que amamentar é mais que um ato fisiológico e biológico, mas sim um evento que abrange sentimentos, emoções e desejos (CRUZ, SEBASTIÃO, 2015).

Nesse sentido deve-se considerar que apesar de comumente o aleitamento estar associado à sucção mamária, ele pode ser desenvolvido de diferentes formas. A prática do aleitamento permite que a oferta de leite humano vá desde a sucção à mama materna até a extração manual de leite (oferecido por seringa ou gavagem) e à translactação (MORAIS; GUIRARDI, MIRANDA, 2020).

\subsection{Fatores que influenciam no desfecho da amamentação em UTIN.}

Mesmo diante de muitos benefícios que o AM proporciona, observa-se que ainda há dificuldades na sua oferta exclusiva durante a internação hospitalar. A amamentação no contexto da prematuridade traz consigo diversas dificuldades, tanto biológicas quanto psicossociais (BEZERRA, et al 2017).

Proporcionando a mãe sentimos ambivalentes, ao vivenciar a alegria da chegada de seu filho, mas também, vivenciar sentimentos de frustação e culpa pela fragilidade do RN (UEMA, et al 2015).

Fisiologicamente, a prematuridade faz com que os recém-nascidos se apresentem imaturos quanto ao controle da sucção, respiração e deglutição, o que muitas vezes leva a dificuldade da manutenção da amamentação. Especificamente na prematuridade amamentar pode tornar- se um ato complexo, exigindo da mãe muita dedicação, além do suporte familiar e profissional (BEZERRA, et al 2017).

É visto que a hospitalização exerce um efeito negativo na amamentação, a percepção materna em relação ao bebê não idealizado faz com que a mãe demore a aceitar essa realidade, além disso, a insegurança de lidar com bebês tão frágeis e delicados as leva a acreditar que não são capazes de amamentar. A partir disso, surge a alta frequência no desmame precoce e a dependência dos bancos de leite (UEMA, et al 2015).

Concomitante ao tempo prolongado de hospitalização e o quadro clínico do bebê surge ainda outro fator que impede à implementação do AM. Em um estudo realizado com seis mães de prematuros hospitalizados em um Hospital percebeu-se que, algumas mães 


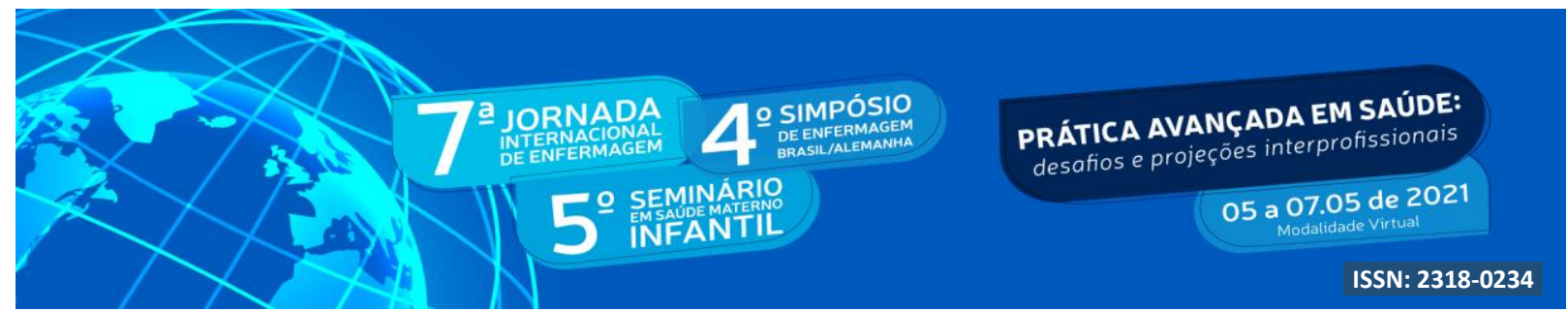

demonstravam-se inseguras em relação à quantidade do leite produzido e à suficiência do mesmo ao bebê (CUNHA, et al 2020).

Além disso, o cansaço materno imposto pela rotina hospitalar parte devido que algumas mães deslocam-se diariamente a UTIN, além de seus afazeres domésticos e cuidados que precisam ser ofertados aos seus outros filhos. Todavia as mães reconhecem a importância da lactação, se empenhando para a manutenção dessa rotina (TRONCO, et al 2015).

No entanto, como resultado desta hospitalização longa e da prematuridade a mãe do neonato é orientada a ordenha do leite, este leite é utilizado para a lactação por meio de sondas e/ou para armazenamento e posterior alimentação do neonato. Essa prática por vezes, gera um sentimento de desânimo visto que é percebida pelas mães como geradora de dor e danos ao seio. Para as mães aleitar está tanto em amamentar diretamente no seio quanto ordenhar, porém, a amamentação ao seio é percebida como uma conquista alcançada pelos esforços conjuntos do binômio mãe e filho (BEZERRA, et al 2017).

Frente a todos esses desafios, propõe-se que esses recém-nascidos devem receber cuidados especiais no que se refere a rotinas e ações dos profissionais de saúde. Sendo necessário dispor de atenção redobrada especialmente na promoção do contato pele a pele, ordenhas periódicas e a oferta desse leite ordenhado quando necessário, além da amamentação diretamente no peito (MACHADO, et al 2019).

\subsection{Os profissionais da equipe de enfermagem e seu papel para manutenção da lactação em UTIN}

No dia a dia de uma UTIN nota-se um cuidado objetivo, rodeado de alta tecnologia com equipamentos especializados, profissionais altamente qualificados e cuidados específicos aos recém-nascidos. Porém mesmo com a disponibilidade desses equipamentos e diversos profissionais percebe-se uma dificuldade em relação à amamentação de prematuros (BEZERRA, et al 2017).

Frente a isso os profissionais exercem o papel de reduzir o medo produzido pelo ambiente desconhecido e oferecer apoio estimulando o contato mais precoce dos pais com seu filho. Dispondo de informações esclarecedoras aos pais, além da manutenção do cuidado no período de internação, e ainda estimular a interação do recém-nascido pré-termo e família (TRONCO, et al 2015). 


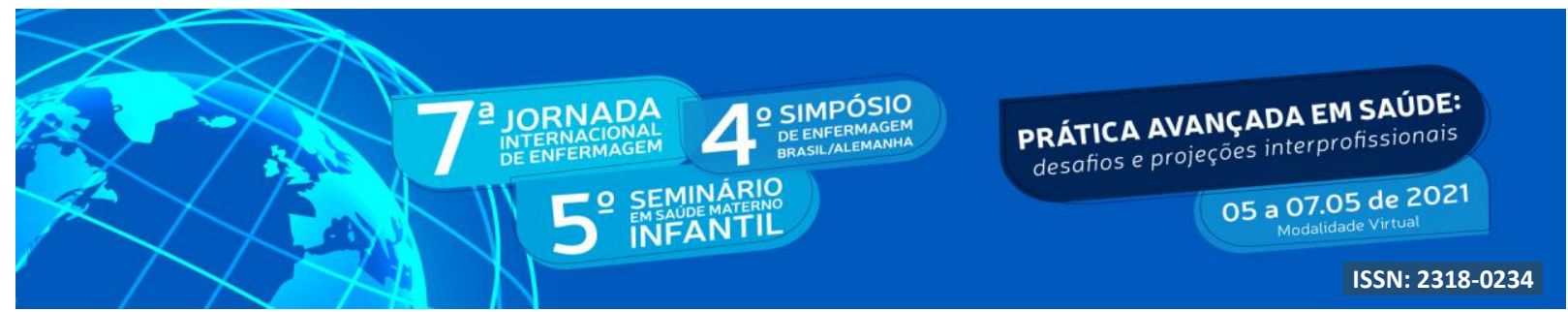

Diversas ações podem ser realizadas para promover o aleitamento, como o estabelecimento de rotinas que possam trazer a promoção e apoio ao aleitamento materno, oferecer orientações às mães de prematuros que não tenham condições de amamentar a proceder a extração manual do leite e orientar quanto ao procedimento, iniciar a dieta (leite materno) via gavagem o mais precocemente possível, assim como promover o contato pele a pele com a mãe sempre que possível, começar a amamentação assim que o quadro clínico do RN permitir, além de mostrar-se presente e disponível à família (MORAIS, GUIRARDI, MIRANDA, 2020)

Quanto a percepção dos profissionais sobre o AM em UTIN, Uema et al (2015) sinaliza que a criação de um ambiente acolhedor e tranquilo para a amamentação serviria de estímulo às mães, que por vezes residem longe do hospital, até mesmo em outros municípios e se deslocam para visitar seus filhos. Além do mais, os profissionais referem à necessidade de dispor de tempo, estímulo e conhecimento de técnicas a fim de se obter um resultado positivo no estímulo ao AM.

Diante da necessidade de lactação os profissionais devem acolher a família, incentivar o vínculo mãe-bebê atentando sempre para os sentimentos expressados pela mãe nesse período, e além disso, desenvolver ações de educação em saúde visando o início precoce da ordenha mamária (TRONCO, et al 2015).

Por fim, ressalta-se que os maiores beneficiados pela amamentação serão a mãe e o bebê, e para isso, os profissionais devem tomar conhecimento que exercem um grande papel. Os problemas frente ao AM pode trazer, no entanto a frustração dos profissionais que estão no cuidado frente aos prematuros, porém quando as ações exercidas mostram o contrário os mesmos terão a certeza do empenho gerado para um desfecho diferente (UEMA, et al 2015)

\section{CONCLUSÃO}

O estudo mostrou o quão é essencial à manutenção da amamentação em uma UTIN, e o quão desafiador é. Notou-se que a amamentação aumenta o vínculo mãe- filho, além de proporcionar à mãe um momento de cuidado e proximidade com seu neonato, o que muitas vezes torna-se quase inexistente frente à rotina de uma unidade intensiva.

Percebeu-se diversas dificuldades de implementação e continuidade do ato de amamentar diante da prematuridade. No entanto, o estudo destaca os profissionais como 


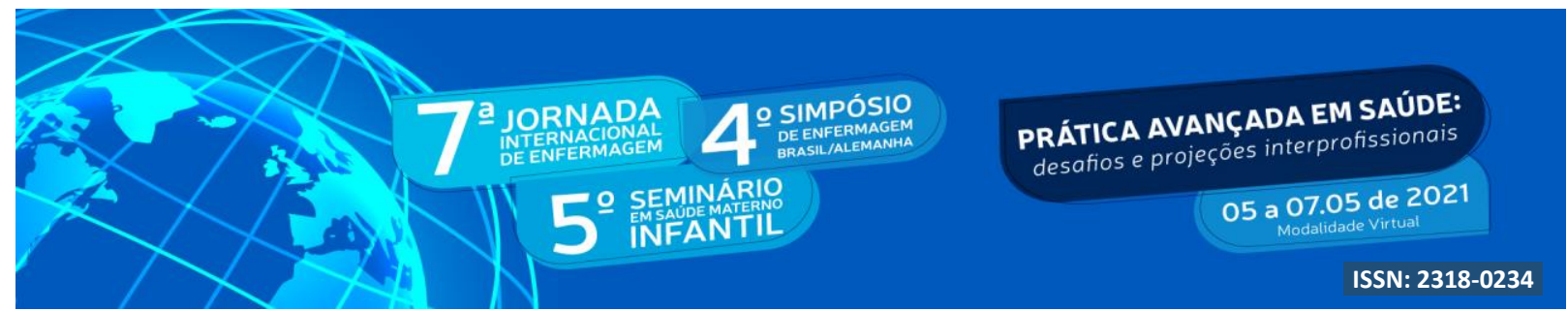

fortes aliados nesse processo, capazes de estimular, sensibilizar e influenciar á mãe e a família, visando um desfecho adequado no processo lactação de um RN.

Frente aos achados deste estudo, acredita-se ser relevante a educação permanente dos profissionais atuantes na UTIN, visando a qualificação da atuação frente à amamentação na prematuridade. Além disso, a educação em saúde emerge como uma necessidade enquanto detentores de conhecimento tanto por parte dos profissionais de enfermagem das UTINs quanto os profissionais que acompanham estas famílias durante todas as consultas de prénatal.

Por fim, ressalta-se a importância da empatia frente às múltiplas situações e desafios enfrentados pelas famílias que estão com seus neonatos hospitalizados. Para isso, é necessário momentos de reflexão acerca da temática, a fim de sensibilizar os profissionais a repensar as suas ações, rever comportamentos e buscar exercer a prática profissional com qualidade.

\section{REFERÊNCIAS}

BEZERRA, M. J. ET AL. Percepção de mães de recém-nascidos prematuros hospitalizados acerca da amamentação. Rev. baiana enferm. v. 31, n.2, p.1-9. 2017.

CRUZ, M.R; SEBASTIÃO, L.T. Amamentação em prematuros: conhecimentos, sentimentos e vivências das mães. Distúrbios Comun. V. 27, n.1, p.76-84. São Paulo. 2015.

CUNHA, G. M; RODRIGUES, F.A; HERBER, S. Aleitamento materno do prematuro em um hospital amigo da criança. Revista Recien. V.10, n. 30, p.168-178. 2020.

LIMA, A. P. E. ET AL. Aleitamento materno exclusivo de prematuros e motivos para sua interrupção no primeiro mês pós-alta hospitalar. Rev Gaúcha Enferm. V. 40, n. 20180406, p. 1-8. 2019.

MACHADO, M. C. H. S. ET AL. Situação do aleitamento materno no primeiro ano de recém-nascidos prematuros tardios: estudo de coorte. Rev. Eletr. Enferm, V. 21, n.52382, p. 1-12. 2019.

MORAIS, A. C; GUIRARDI, S. N; MIRANDA, J. O. F. Práticas de aleitamento materno em unidade de terapia intensiva neonatal. Rev baiana enferm. V. 34, n.35643, p. 1-11. 2020.

TRONCO, C.S. ET AL. Manutenção da lactação de recém-nascido pré-termo: rotina assistencial, relação mãe-filho e apoio. Escola Anna Nery. V. 19, n.4, p.635-40. Out/Dez. 2015. 


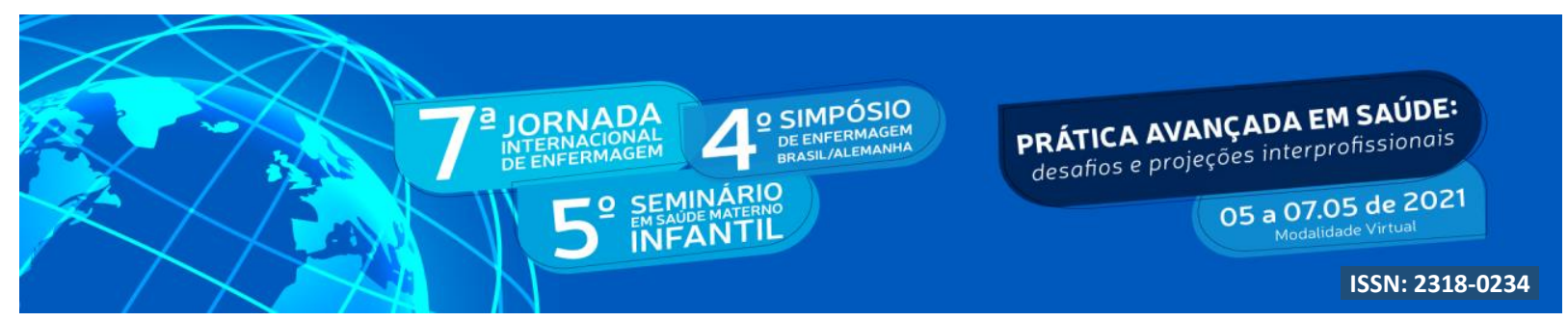

UEMA, R. T. B. ET AL. Insucesso na amamentação do prematuro: alegações da equipe. Rev. Semina: Ciências Biológicas e da Saúde. V. 36, n. 1, p. 199-208. 2015. 